\title{
The Effectiveness of Skill-Based Intervention for Female Victims of Intimate Partner Violence: A Critical Review
}

\author{
Fong Lopaschuk ${ }^{1}$, Cary A. Brown ${ }^{2}$ \\ ${ }^{1}$ Crisis Response Team, Alberta Health Services, Edmonton, Canada \\ ${ }^{2}$ Faculty of Rehabilitation Medicine, University of Alberta, Edmonton, Canada \\ Email: cary.brown@ualberta.ca
}

Received January $25^{\text {th }}$, 2012; revised February $27^{\text {th }}$, 2012; accepted March $11^{\text {th }}, 2012$

\begin{abstract}
Intimate Partner Violence (IPV) is associated with limitations in Activities of Daily Living (ADL). This paper critically reviews the methodological quality of evidence for life skills interventions provided for women who have experienced IPV. A comprehensive search was carried out for all relevant studies and the McMaster critical appraisal tools used to determine methodological quality of selected articles. Three studies met the inclusion criteria for the review. Findings indicate there is limited evidence available to inform practitioners as to benefits or weaknesses of current life skills interventions. IPV has serious individual and social consequences and methodologically rigorous research is urgently needed.
\end{abstract}

Keywords: Intimate Partner Violence; Intervention; Activities of Daily Living (ADL); Spousal Abuse; Critical Review

\section{Introduction}

Intimate Partner Violence (IPV) is a recognized social epidemic with devastating individual and societal consequences (PHAC, 2009). IPV is a term used to depict violence occurring between people in intimate relationships. While there is no standard definition of IPV, it is generally agreed IPV includes physical aggression, psychological abuse, economic abuse, forced intercourse and other forms of sexual coercion resulting in physical, psychological or sexual harm (PHAC, 2009; Statistics Canada, 1995; Statistics Canada, 2008). Unlike other types of violence, the people involved in intimate relationships may share children and economic ties in addition to emotional attachments.

IPV not only directly affects victims' emotional and physical well-being but also affects the children exposed and in turn, has a far-reaching impact on the society as a whole. In almost $40 \%$ of domestic abuse cases, children are exposed to violence against their mothers, often of a very serious nature (Statistics Canada, 2008). Children of mothers who are exposed to IPV experience a variety of negative emotional and/or somatic symptoms often accompanied by multiple impairments in their own interpersonal relationships, as well as in many other aspects of their lives (Suglia, Enlow, Kullowatz, \& Wright, 2009). These impairments include distress that mirrors their mothers' symptoms (Lang \& Stover, 2008), higher vulnerability to abuse (Taylor, Guterman, Lee, \& Rathouz, 2009), and maladaptive peer relations (Katz, Hunterm, \& Klowden, 2008). Additionally, of significant concern is that research demonstrates exposure to parental violence is associated with violent interactions in adulthood (Ireland \& Smith, 2009).

While IPV can be perpetrated by both men and women, in both homosexual and heterosexual relationships, women are particularly vulnerable to abuse, especially women with disabilities (Forte, Cohen, DuMont, Hyman, \& Omans, 2005). As opposed to men, who are more likely to be attacked by a stranger or an acquaintance, violence against women is frequently per- petrated by a husband or an intimate male partner (Forte et al., 2005). In Canada, women are consistently more vulnerable to IPV across all provinces and territories, accounting for $83 \%$ of all reported cases of domestic violence (Statistics Canada, 2008). Female victims of IPV sustain more frequent and more severe injuries and are more likely to fear for their lives than their male counterparts (Statistics Canada, 2005). In the cases of violence analyzed in Mechanic, Weaver and Resick’s (2008) study of 362 battered women from residential and non-residential community agencies, nearly half of the sample reported being hit repeatedly on the head, or sustaining injuries resulting in loss of consciousness. Nearly three quarters of the women in this study reported strangulation. It follows that for some women this repeated head and neck trauma and partial or complete loss of consciousness may have enduring neuropsychological and psychological sequels, such as diminished cognitive functioning and post-traumatic stress disorder.

Given the sensitive and personal nature of this type of violence, victims do not always report all incidents of abuse (Spangaro, Zwi, Poulos, \& Man, 2012), and the majority of violence experienced in spousal relationships is believed to be recurrent in nature. Half of self-reported IPV victims state that the violence has occurred on more than one occasion, and only $27 \%$ of victims report the incident to police (Statistics Canada, 2005).

While not all incidences of IPV are reported, IPV represents a reality for many couples around the world. According to the World Health Organization, $10 \%$ to $69 \%$ of adult women report having been physically abused by a partner at least once in their lives, while $6 \%$ to $47 \%$ report sexual abuse in their intimate relationships (Krug, Dahlberg, Mercy, Zwi, \& Lorano, 2002). In Canada, over 38,000 incidents of spousal violence were reported across the country in 2006, representing $15 \%$ of all policereported violent incidents (Statistics Canada, 2008).

The WHO's multi-country report on IPV, a study consisting of 24,000 women in ten countries, found that women who experienced physical or sexual abuse, or both, were significantly 
more likely to report poor or very poor health than women who had not experienced IPV (Krug et al., 2002). Long-term negative health consequences for women exposed to IPV are documented extensively in the literature (Bonomi, Anderson, Rivara, \& Thompson, 2007; Campbell, 2002; Forte et al., 2005; McNutt, Carlson, Persaud, \& Postmus, 2002; Plichta, 2004).

Victims of IPV experience a range of physical problems. In the Canadian National Survey on Violence against Women, $45 \%$ of wife assault cases resulted in physical injury, the most frequent type being bruises (90\%), followed by burns (33\%), and broken bones (12\%), with almost $10 \%$ of injured women reporting internal injuries and miscarriages (Statistics Canada 1994). Gynecological problems are reported to be the most consistent, enduring, and largest physical health difference between battered and non-battered women (Campbell, 2002). Abused women have $50 \%$ to $70 \%$ increase in gynecological, central nervous system and stress-related problems, such as sexually transmitted diseases, vaginal bleeding, vaginal infections, pelvic pain, painful intercourse, urinary tract infections, headaches, back pain, appetite loss, abdominal pain, and digestive problems (Campbell, Jones, Dienemann, Kub, Schollenberger, O'Campo et al., 2002). It is not surprising that IPV is also related to a signifycantly high user rate for healthcare and social services, unmet needs for medical care, and to increased medical costs (Plichta 2004, Wood, Hall, Campbell, \& Angott, 2008, Wuest, MerrittGray, Ford-Gilboe, Lent, Varcoe, \& Campbell, 2008). Women exposed to IPV also experience long term mental health conesquences such as depression and posttraumatic stress disorders (Bonomi et al., 2007). In addition, in all settings, victims of IPV report significantly higher levels of emotional distress, thoughts of suicide and suicide attempts than women who have never experienced IPV (Krug et al., 2002).

IPV victims' occupational performance is adversely affected by their poor mental and physical health. Occupational performance is generally defined as the ability to engage in activities of everyday living in a manner that the individual perceives as both successful and satisfying (Brown, 2009; CAOT, 2007). Victims of IPV experience more activities limitations than average Canadian women (Krug et al., 2002), due to the fact that they are more likely to have problems walking, and because they often experience memory loss, dizziness and chronic pain (Wuest et al., 2008).

As a result of activities limitations, IPV adversely impacts numerous occupational performance areas in the Instrumental Activities of Daily Living (IADL). IADL differs significantly from Basic Activities of Daily Living (BADL), which focuses primarily on self-care tasks such as feeding and dressing: IADL is defined as activities requiring cognitive and physical skills for independent living, such as budgeting and parenting (CAOT, 2007). As performance in IADL decreases for victims of IPV, their roles as mothers and workers are the most notably affected. Poor health and chronic pain in survivors of IPV interfere with employment and child rearing (Wuest et al., 2008). Mothers currently experiencing IPV, or having experienced IPV in the past, are significantly and definitively associated with maternal depression (Casanueva, Foshee, \& Barth, 2005; Hazen, Connelly, Kelleher, Barth \& Landsverk 2006). There is a positive association between children whose mothers experience severe intimate partner violence and these children's injuries, largely due to a lack of adult supervision, resulting in use of emergency room facilities (Casaneueva et al., 2005). These children are also more likely to experience maltreatment (Ireland \& Smith,
2009). However, maternal ability to maintain positive care giving processes in an abusive context may buffer the effects of domestic violence on children. These findings underline that the difficulties inherent to the occupation of motherhood are exacerbated for victims of IPV (Krane \& Davies, 2007) and provide insight as to why women who experience IPV struggle with the parenting skills that are needed for a child's physical and emotional well-being and development. Some research suggests the best way to promote positive health in toddlers may be to help their mothers deal with relationships involving IPV (Suglia et al., 2009).

Due to the traumatic nature of intimate partner violence and its negative impact on the occupational performance aspects required for successful independent living skills, women experiencing IPV are at an increased risk of becoming homeless and/or dependent on their abusers (Helfrich \& Aviles, 2001). The results from Kimerling, Alvarez, Pavao, Mack, Smith, and Baumrind's (2009) study of 6698 battered women indicated substantial rates of unemployment. IPV is also linked to poor employment stability, and a high use of welfare (Busch \& Wolfer, 2002; Kimmerling et al., 2009). Researchers found that both victims of IPV and emergency shelter staff members considered that increasing abused women's IADL skills, (such as seeking and retaining employment, parenting, budgeting, time management, social navigation skills to locate community resources for housing, child care services and employment services) were the most urgent intervention priority (Gorde, Helfrich, \& Finlayson, 2004).

While there has been an explosion of studies in the past few decades describing the short and long term harm of IPV resulting in numerous endeavors to assist victims of family violence, there is a paucity of outcome research evaluating the effectiveness of these interventions (Plichta, 2004; Wathen \& MacMillan, 2003). Most, if not all, interventions appear to have occurred in the absence of scientific evidence on the benefits to be gained by the use of these programs (Wathern \& MacMillan, 2003). According to Plichta (2004) there are few, if any, studies in the interrelated fields of health, occupational therapy, dentistry, health care administration, and pharmacy on the effectiveness of services being provided to victims of IPV. A scientific review of interventions for violence against women revealed that no studies have evaluated the usefulness of screening to reduce violence or improve women's health, nor were there any studies evaluating the success rate of shelter stay as a means to decrease incidences of violence (Helfrich, et al., 2006). While a systematic review (Helfrich \& Rivera, 2006) revealed advocacy, career counseling plus conscious awareness, cognitive behavioral counseling, cognitive trauma therapy, peer support groups, and safety planning as being beneficial for female victims of IPV, little information is available as to the efficacy and relevance of life skills interventions for this population (Gorde et al., 2004; Helfrich et al., 2006; Helfrich \& Rivera, 2006).

It is apparent that community-based service providers have both an obligation and the opportunity to address a variety of important life skills for victims of IPV Helfrich \& Aviles, 2001). Occupational therapy is an example of a profession particularly suited to provide intervention to increase occupational performance, as occupational therapists are trained in assessing and providing therapeutic interventions to address individuals coping skills for the challenges that arise from the dynamic interactions between peoples and their environment. Given the profession's core philosophy of promoting self-efficacy, life 
skills for self-management, and social and occupational justice, occupational therapy have the capacity to assume not only direct therapeutic intervention but also activism and advocacy activities on behalf of those who are vulnerable and marginalized (Cage, 2007).

Occupational therapy has a social vision with its core mission to enable occupations through therapeutic use of activity in promotion of function. Occupational therapists specialize in promoting engagement in daily life activities, and can play a vital role in increasing occupational participation (CAOT, 2007). IPV hinders victims from engaging in daily activities in a healthy, competent and satisfying manner. Occupational Therapists can play a role in restoring and enhancing the ability of those women experiencing IVP to participate in their chosen ADL by focusing on facilitating the acquisition of skills for healthy, independent lifestyles and the subsequent improvement of quality of life (Javaherian, Underwood, \& Delany, 2007; Olivas-De La, 2008).

Recognizing that outcome assessment of interventions is necessary in order to deliver quality programming, research and theory is beginning to emerge to assist those who experience IPV to develop or restore their abilities to engage in daily life occupations. One example is Helfrich and Avile's (2001) recommendation for a theory-based outline of assessment and treatment procedures for occupational therapists working with those who have experienced IPV.

Our background search revealed a gap between the theorybased discussion of IPV and the apparent lack of findings for intervention results. To better guide both best-practice and future research, a systematic review of all outcome studies to clarify the benefits of IADL intervention for victims of IP is required. With that goal, this paper provides a critical review of the different IADL treatment program research to enhance women's participation in their instrumental activities of daily living.

\section{Research Question and Aim}

The research question this paper intends to address is the following: What is the strength of evidence in favor of ADL skills intervention for female victims of heterosexual IPV? The aim of this paper is to analyze studies concerning interventions geared towards increasing ADL skills in the areas of self-care, leisure and productivity.

\section{Methods}

The first author (FL) carried out a Critical Literature Review (CR) following the standardized approach defined by Law and MacDermid (2008). Critical Reviews following these protocols include qualitative, quantitative and mixed methods studies. This approach was selected so as to ensure the identification and assessment of the range of relevant research methodologies. This broad scoping is important when addressing complex, non-linear social and health-care issues like IPV directed at Canadian women. The McMaster Protocols were first published on-line in 1998 and updated in 2008 (Law, 2008). They are extensively used within rehabilitation research and are now translated into four languages. The Critical Review process result in a systematic synthesis of published research findings on ADL interventions based on an explicit search strategy and evaluation of this literature against quality indicators as identified in the McMaster protocols (Law, 2008). The steps are outlined below.

\section{Search Strategy}

The following databases were searched: AMED (Ovid), CINAHL (EBSCO), MEDLINE (Ovid), PsychInfo (Ovid), OTDbase and OTSeeker. A researcher in the field of Family Studies and a medical librarian were consulted to validate the acceptability and the comprehensiveness of the search strategies. Details of the search are available from the corresponding author.

\section{Inclusion and Exclusion Criteria}

For this review, IPV was defined as the physical and psychological abuse of women perpetrated by their male partners. This Critical Review focuses on the effectiveness of intervenetions to enhance engagement in ADL. Therefore, publications were included if they had been peer-reviewed and reported on a therapeutic intervention that addressed ADL interventions in the area of self-care, leisure or productivity for women who had experienced IPV. The Critical Review included only outcomebased research. All articles, irrespective of research design, were included. Studies on substance abuse; prevention of IPV, programs addressing sexual assault by strangers or acquaintances, childhood sexual abuse, or studies concerning interventions not geared towards increasing ADL skills were excluded. The research question focused on outcomes with clinically relevance to healthcare providers, such as occupational therapists, who specialize in ADL and increasing the occupational performance of women who have experienced IPV. Therefore, studies that evaluate specialized psychological intervention, psychoanalytic therapy or counseling alone were excluded.

\section{Study Selection}

The preliminary search resulted in 494 citations. Review of the abstracts and application of the exclusion/inclusion criteria resulted in four articles. The first author (FL), examined the full text of these and manually searched the reference lists of these articles. Subsequently three articles (Gutman et al., 2004; Helfrich \& Avileo, 2001; McFarlane et al., 2002). were retained for full review and critical analysis (Table 1). The most frequent reason for the exclusion of a given paper was the focus on topics other than interventions for female victims of IPV, such as drug abuse, childhood sexual abuse, and interventions for the perpetrators of IPV.

\section{Quality Rating}

The Critical Review protocols (Law, 2008) rate the research quality of individual studies and are based on a determined set of operational parameters including the following 12 categories: study purpose, literature, design, design types, appropriateness of study design, biases, sample, outcomes, intervention, results, drop outs and conclusions and clinical implications. The Jadad scoring system was also used to assess the quality of randomization achieved in the one randomized control trial (RCT) retrieved in the search (Jadad, Moore, Carrolee, Jenkison, Reynolds et al., 1996). The Jadad Scale is a simple, valid, reliable instrument that assists in critical evaluation of randomization in RCTs (Jadad et al., 1996).

\section{Findings}

Systematic review of the literature revealed a paucity of material regarding ADL interventions for IPV victims. Of the three 
Table 1.

Descriptions of studies included in the review of ADL interventions for victims of IPV.

\begin{tabular}{|c|c|c|c|c|}
\hline Study & Aims & Intervention & Outcome Assessments & Outcome \\
\hline $\begin{array}{l}\text { Gutman } \\
\text { et al. (2004) }\end{array}$ & $\begin{array}{l}\text { Assess effectiveness of an } \\
\text { intervention for women experiencing } \\
\text { IPV and/or homelessness, whose } \\
\text { cognitive impairments may have } \\
\text { contributed to their inability to } \\
\text { leave an abusive situation }\end{array}$ & $\begin{array}{l}1 \mathrm{hr} / \text { week for six months } \\
\text { via group and individual 1:1 } \\
\text { sessions on } 12 \text { IADL areas }\end{array}$ & $\begin{array}{l}\text { GAS scores, pre-post tests } \\
\text { chart review, OT students } \\
\text { logs, acceptability question } \\
\text { naires }\end{array}$ & $\begin{array}{l}99 \% \text { of the participants expressed a high } \\
\text { degree of satisfaction regarding the } \\
\text { interventions. All but one participant } \\
\text { achieved expected outcome or greater in } \\
\text { all intervention areas. }\end{array}$ \\
\hline $\begin{array}{l}\text { Helfrich } \\
\text { et al. (2006) }\end{array}$ & $\begin{array}{l}\text { Enhance employment knowledge } \\
\text { and skills in a group of IPV } \\
\text { survivors residing in a shelter }\end{array}$ & $\begin{array}{l}8 \text { sessions in } 4 \text { weeks via } \\
\text { group and 1:1 sessions } \\
\text { on vocational skills }\end{array}$ & $\begin{array}{l}\text { Individual knowledge base } \\
\text { and client satisfactions }\end{array}$ & $\begin{array}{l}\text { Clinical intervention was effective in } \\
\text { providing opportunity to develop skills } \\
\text { critical to obtain and maintain employment. } \\
\text { Article did not address results from weekly } \\
\text { quizzes and pre/post Ax designed to evaluate } \\
\text { information that had been reviewed. }\end{array}$ \\
\hline $\begin{array}{l}\text { McFarlane } \\
\text { et al. (2002) }\end{array}$ & $\begin{array}{l}\text { Increase the number of "safety } \\
\text { promoting" behaviours practiced } \\
\text { by abused women. }\end{array}$ & $\begin{array}{l}\text { Six safety intervention phone } \\
\text { calls, first within } 48 \text { - } 72 \text { hrs } \\
\text { of initial visit, then remaining } \\
\text { calls at } 1,2,3,5,8 \text { weeks, with } \\
\text { discussion of safety behaviour } \\
\text { strategies }\end{array}$ & $\begin{array}{l}\text { Safety Behaviour Checklist } \\
\text { Checklist completed at } \\
\text { each phone call and at } 3 \\
\text { and } 6 \text { month follow up } \\
\text { phone calls to assess women } \\
\text { on the use of safety behaviours }\end{array}$ & $\begin{array}{l}\text { Participant from intervention group practiced } \\
\text { significantly higher number of safety } \\
\text { behaviours at } 3 \text { and } 6 \text { months. }\end{array}$ \\
\hline
\end{tabular}

papers dealing specifically with increasing ADL skills, Helfrich and Rivera (2006) focused on vocational skills. Gutman et al. (2004) addressed twelve different IADL interventions, includeing, safety planning, vocational skills, practical life skills and controlling risky behaviours, and McFarlane et al. (2002) focused on safety behaviours. Only Gutman et al. (2004) explored leisure as a topic for ADL intervention. Table 2 contain a descriptive summary of each study included in the critical review and Table 1 presents a descriptive summary and Table 2 presents the comparative findings of the quality critique for each of the three studies.

Study 1: Gutman et al.'s (2004) study of 26 women employed a cohort design and evaluated a program that addresses possible cognitive deficits in women who had experienced IPV as compared to what Gutman et al identified as traditional treatment based on a psychological perspective. Their study utilizes a mixedmethod design and a convenience sample comprised of 26 women, who all reported having a disability. The intervention targeted ADL needs congruent with those highlighted in the literature and also focused on the participants' needs as identified in initial assessments. The specific ADL skills intervention focused on the following areas: safety planning, drug and alcohol awareness, safe sex practices, assertiveness and advocacy skill training, anger management, stress management, boundary establishment and limit-setting, vocational and educational skill training, money management, housing application, leisure exploration and hygiene, medication routine and nutrition. Participants received intervention for one hour per week over the course of 6 months via group and one-to-one sessions provided by four OT students who were supervised by two OTs.

An analysis of the strengths and weaknesses of the study reveals several methodological shortcomings. Specifically, co-interventions may have been present because some of the participants were living in local shelters, where they may have received other similar life skills programs. Also, the assessment tools utilized to determine if participants increased their knowledge were not validated nor psychometrically tested for reliability and validity. Strengths of this research include the utilization of a mixed-method approach with qualitative and quantitative data collection, which increases internal validity via methodological triangulation. Triangulation of multiple data sources (background
Table 2.

Methodological quality indicators for reviewed studies.

\begin{tabular}{|c|c|c|c|}
\hline & $\begin{array}{c}\text { Gutman et al. } \\
\text { (2004) }\end{array}$ & $\begin{array}{c}\text { Helfrich \& } \\
\text { Avileo (2001) }\end{array}$ & $\begin{array}{c}\text { McFarlane et al } \\
\text { (2002) }\end{array}$ \\
\hline \multicolumn{4}{|l|}{ Study purpose: } \\
\hline Stated clearly? & yes & yes & yes \\
\hline $\begin{array}{l}\text { Relevant literature } \\
\text { reviewed? }\end{array}$ & yes & yes & yes \\
\hline \multicolumn{4}{|l|}{ Design: } \\
\hline Type? & BA & BA & RCT \\
\hline Appropriate? & yes & yes & yes \\
\hline \multicolumn{4}{|l|}{ Sample/Methods: } \\
\hline Sample detailed? & yes & yes & yes \\
\hline Sample justified? & yes & no & yes \\
\hline Jadad Score & N/A & N/A & 3 \\
\hline \multicolumn{4}{|l|}{ Outcomes: } \\
\hline Reliable? & yes & no & yes \\
\hline Valid? & yes & no & yes \\
\hline \multicolumn{4}{|l|}{ Intervention: } \\
\hline Described in detail? & yes & yes & yes \\
\hline Contamination avoided? & N/A & N/A & no \\
\hline Co-intervention avoided? & no & no & no \\
\hline \multicolumn{4}{|l|}{ Results: } \\
\hline Reported with Stat. Sig.? & yes & no & yes \\
\hline Analysis appropriate? & yes & no & yes \\
\hline $\begin{array}{l}\text { Clinical importance } \\
\text { reported? }\end{array}$ & yes & yes & yes \\
\hline Drop-outs reported? & no & no & yes \\
\hline \multicolumn{4}{|l|}{ Conclusions: } \\
\hline Conclusions appropriate? & Yes & Unclear & Yes \\
\hline Methodological quality & weak & weak & weak \\
\hline
\end{tabular}

literature, the thematic analysis and the data mining results) strengthens the identification of patterns within the phenomena being examined (Denzin \& Lincoln, 1994).

Gutman et al. (2004) utilized the Goal Attainment Scales 
(GAS) (Kiresuk \& Sherman, 1968) for pre- and post-tests, chart reviews, occupational therapy student logs and a review of progress notes in the medical record. The use of GAS was appropriate, because this criterion-referenced measure allows the researcher to quantify specific changes in behaviours and to detect small changes over time (Ottenbacher \& Cusick, 1993). Statistical analysis was also appropriate for this study. Gutman et al. (2004) performed a $t$ test by converting the raw scores from the GAS into a standardized $T$ score. The $t$ test is useful because it can be applied to a small sample size in order to infer treatment effectiveness (Law, 2008). The results suggest a high level of success in attaining goals. Based on the methodologies used and valid outcome measures, this study has an acceptable level of internal validity.

Study 2: Helfrich and Rivera (2006) studied a vocational program for women who had left relationships with IPV for the safety of an emergency shelter. Their life skills intervention focused on enhancing employment knowledge and skills. The researchers utilizing a before and after research design and pre and post self-assessment.

The sample consisted of only 11 women, and the process of sample recruitment and the consent process are not clear from the report. It also appears that participants were not necessarily aware they were involved in a research study as opposed to the routine interventions offered at the shelter. Additionally, the sample size was not justified, no power calculation was provided, and only anecdotal results were reported with no statistical analysis provided. Intervention consisted of 8 sessions of 4 weeks in duration, via group and one-to-one sessions. Transparency in the intervention process was poor. According to Siebes et al. (2007), transparency is comprised of both openness and accountability, and all parties involved should have been clear on the identified occupational performance issues, the treatment process, activities and goals. Upon program completion, participants voiced frustration with the facilitators' inability to secure employment for them. Attendance to the program was inconsistent and attrition was not addressed. Other possible methodological shortcomings were that while pre/post-test selfassessments and weekly quizzes were administered to determine knowledge retention, validity of these assessments and quizzes was not presented, and no standardized, quantifiable measures of change were employed. Secondly, statistical analysis of these outcome measures was not conducted to infer treatment effecttiveness, nor was this discussed in the results section. Additionally, the group leader and the recorder alternated weekly, creating possible bias resulting from experimenter expectancies and attention bias. Finally, co-intervention was possible since participants likely attended other required programs at the shelter on other aspects of life skills. No mention of control for this was made in the paper.

While it may be feasible to repeat these types of intervenetions in other primary care settings, Helfrich and Rivera's report raises the point that IPV victims often require a multidimensional approach from the community, as women living in emergency shelter may have different needs than women living in transitional housing. While this study concluded that the intervention was effective, the limitations presented in the research methods reduce the trustworthiness of this article.

Study 3: McFarlane et al., (2002) conducted a Randomized Control Trial (RCT) to determine the effectiveness of a telephone intervention promoting safety behaviours for victims of IPV. This paper utilizes two conceptual models to guide an 18- month clinical trial. A power analysis was conducted to ensure statistical power, and 150 participants agreed to participate in this study. A systematic allocation of 75 women in the control group and 75 women in the treatment group was utilized to prevent sampling bias. When the researchers calculated descriptive statistics for study variables they found no significant demographic differences between groups with the exception of age, which they then treated as a covariate in further analysis where they found age did not to affect the outcome measure. This study also employs Safety Behaviour Checklist, a measure previously used by the study authors (McFarlane \& Parker, 1994). The author stated the measure had been tested and proven effective, but no psychometric data were reported.

Six intervention phone calls were provided for the treatment group, where the investigator discussed specific safety-promoting behaviours. Paired $t$ test and Repeated Measures of Analysis of Variance were conducted to calculate any differences in knowledge retention between the two study groups. This paper reported that participants from the intervention group practiced a significantly higher number of safety behaviours after 3 and 6 months. There was an excellent retention rate: only one participant from the control group was unable to complete the program due to suicide. This study scored a $3 / 5$ on the Jadad scale as neither the participants, nor those workers providing intervention, were blind to the group allocation.

\section{Discussion}

In general all three studies were methodologically weak, nonreplicable, and the results therefore not strongly reliable. Also, none of the studies evaluated the potential harms of the interventions provided. Consequently it remains unclear how these intervention affect women who may still be involved in violent relationships.

According to Gill and Brown (2009: p. 214), "Rehabilitation has a theoretical grounding directly addressing people's need for empowerment to assume active self-management of their health and control over their living environment.” Much has been learned about the epidemiology of violence against women, but information on evidence-based approaches in developing rehabilitation intervention programs is still severely lacking. Healthcare providers who focus on function and daily activity, like occupational therapists for example, can play a critical role for victims of IPV by enhancing their ability to perform ADL. Researchers have identified the promotion of women's emotional and physical functioning as key in supporting women in the development of coping and leaving strategies (Cage, 2007). Further high level, high quality research into specific skill interventions programs concerning ADL areas, not limited to parenting, budgeting and vocational skills is recommended. Other areas of research worth exploring include education and awareness training for occupational therapists and students, so they can better address occupational dysfunction for victims of IPV.

Although there is a scarcity of studies concerning the effectiveness of ADL intervention for victims of IPV, this critical review demonstrates that there are some pioneering studies available. The preceding critique and discussion synthesizes key findings from the literature concerning the interventions of safety planning and vocational counseling. These two areas of intervention, although being the most widely addressed in the studies reviewed, demonstrated limited evidence of effectiveness. 
This finding is also consistent with Gorde et al.'s (2004) study, which concludes that female survivors prioritize independence and productivity over other concerns. The following discussion gives more detailed examples of how these two areas were operationalized within the studies.

\section{Intervention 1: Safety Planning}

Safety planning was a key component in both the Gutman et al. (2004) and MacFarlane et al. (2002) studies. Participants were taught strategies such as identifying signs of escalating violence, safe locations, and important documents to take when fleeing abuse. Other strategies included hiding money, maintaining a safety bag in a discreet location, developing a code for use with family and friends to signal need for assistance, and removing and disposing of weapons in the house.

\section{Intervention 2: Vocational Counseling}

Vocational counseling was discussed in Gutman et al. (2004) and Helfrich and Rivera's (2006) papers. Topics in this area generally include identifying possible careers of interest, exploring vocational training and/or educational options, resume and cover letter writing and interview preparation.

\section{Conclusion}

Research exploring the effectiveness of ADL interventions for victims of IPV is promising but very much in its early stages. This structured scoping and critical analysis of existing outcome studies demonstrates that current programs in the community operate despite the current lack of methodologically sound and reliable evidence of their effectiveness. The following are the key messages from the critical review findings:

- There is an urgent need for higher quality research on the usefulness of skills intervention for victims of IPV.

- There is some limited evidence suggesting that interventions in the areas of self-care and productivity are useful skills and knowledge for victims of IPV. These findings are consistent with Kimerling et al.'s (2009), conclusion that further studies on employment programs for victims of IPV are warranted.

The background literature demonstrates that healthcare providers have theoretical constructs (like occupational performance) that could address life skills and ADL issues for women who are affected by the trauma of domestic violence (Johnston, Adams, \& Helfrich, 2001). However, existing evidence is very scant and of insufficient quality to evaluate the effectiveness of ADL interventions for victims of IPV. Of all relevant studies reviewed, only McFarlane et al.'s (2002) study approached moderate methodological quality and potentially demonstrated a positive relationship between ADL training and positive outcomes. The other studies reviewed offer some limited evidence suggesting that other IADL interventions may also demonstrate successful outcomes for women who experience IPV. It is important to reiterate that there is an urgent need for high quality research on the usefulness of life skills interventions for victims of IPV. Clinicians should carefully scrutinize the evidence-base available and work with researchers to build methodologically robust research to inform practice when serving the victims of IPV.

\section{REFERENCES}

Bonomi, A. E., Anderson, M. L., Rivara, F. P., \& Thompson, R. S. (2007). Health outcomes in women with physical and sexual intimate partner violence exposure. Journal of Women's Health, 16, 987-997. doi:10.1089/jwh.2006.0239

Brown, C. (2009). Functional assessment and intervention in occupational therapy. Psychiatric Rehabilitation Journal, 32, 162-170. doi:10.2975/32.3.2009.162-170

Busch, N. B., \& Wolfer, T. A. (2002). Battered women speak out: Welfare reform and their decisions to disclose. Violence against Women, 8, 566-584. doi:10.1177/107780102400388434

Cage, A. (2007). Occupational therapy with women and children survivors of domestic violence: Are we fulfilling our activist heritage? A review of the literature. British Journal of Occupational Therapy, 70, 192-198

Campbell, J. C. (2002). Health consequences of intimate partner violence. Lancet, 359, 1331-1336. doi:10.1016/S0140-6736(02)08336-8

Campbell, J., Jones, A. S., Dienemann, J., Kub, J., Schollenberger, J., O'Campo, P. et al. (2002). Intimate partner violence and physical health consequences. Archives of Internal Medicine, 162, 1157-1163. doi:10.1001/archinte.162.10.1157

Canadian Association of Occupational Therapists. (2007). Enabling occupation II: Advancing an occupational therapy vision for health, well-being, \& justice through occupation. Ottawa, Ontario: CAOT Publication ACE

Casanueva, C., Foshee, V. A., \& Barth, R. P. (2005). Intimate partner violence as a risk factor for children's use of emergency room and injuries. Children and Youth Services Review, 27, 1123-1242. doi:10.1016/j.childyouth.2005.04.006

Casteel, C., \& Sadowski, L. (2009). What are the effects of interventions initiated by healthcare professionals aimed at women victims of intimate partner violence? Clinical Evidence, 2, 1013-1030.

Denzin, N. K., \& Y. S. Lincoln (1994). Handbook of qualitative research. London, Sage Publications.

Forte, T., Cohen, M. M., Du Mont, J., Hyman, I., \& Omans, S. (2005). Psychological and physical sequelae of intimate partner violence among women with limitations in their activities of daily living. Archives of Women's Mental Health, 8, 248-256. doi:10.1007/s00737-005-0093-9

Gill, J. R., \& Brown, C. A. (2009). A structured review of the evidence for pacing as a chronic pain intervention. European Journal of Pain, 13, 214-216. doi:10.1016/j.ejpain.2008.03.011

Gorde, M. W., Helfrich, C. A., \& Finlayson, M. L. (2004). Trauma symptoms and life skill needs of domestic violence victims. Journal of Interpersonal Violence, 19, 691-708. doi:10.1177/0886260504263871

Gutman, S. A., Diamond, H., Holness-Parchment, S., Brandofino, D. N., Pacheco, D. G., Jolly-Edouard, M. et al. (2004). Enhancing independence in women experiencing domestic violence and possible brain injury: An assessment of an occupational therapy intervention. Occupational Therapy in Mental Health, 20, 49-79. doi:10.1300/J004v20n01 03

Hazen, A. L., Connelly, C. D., Kelleher, K. J., Barth, R. P., \& Landsverk, J. A. (2006). Female caregivers' experiences with intimate partner violence and behavior problems in children investigated as victims of maltreatment. Pediatrics, 117, 99-109. doi:10.1542/peds.2004-2542

Helfrich, C. A., \& Aviles, A. (2001). Occupational therapy’s role with victims of domestic violence: Assessment and intervention. Occupational Therapy in Mental Health, 16, 53-57. doi:10.1300/J004v16n03_04

Helfrich, C. A., Aviles, A. M., Badiani, C., Walens, D., \& Sabol, P. (2006). Life skill interventions with homeless youth, domestic violence victims and adults with mental illness. Occupational Therapy in Health Care, 20, 189-207. 0.

Helfrich, C. A., \& Rivera, Y. (2006). Employment skills and domestic violence survivors: A shelter-based intervention. Occupational Therapy in Mental Health, 22, 33-48. doi:10.1300/J004v22n01_03

Ireland, T. O., \& Smith, C. A. (2009). Living in partner-violent families: Developmental links to antisocial behavior and relationship violence. 
Journal of Youth \& Adolescence, 38, 323-339.

doi:10.1007/s10964-008-9347-y

Jadad, A. R., Moore, R. A., Carroll, D., Jenkinson, C., Reynolds, D. J., Gavaghan, D. J. et al. (1996). Assessing the quality of reports of randomized clinical trials: Is blinding necessary? Controlled Clinical Trials, 17, 1-12. doi:10.1016/0197-2456(95)00134-4

Javaherian, H. A., Underwood, R. T., \& DeLany, J. V. (2007). Occupational therapy services for individuals who have experienced domestic violence (statement). American Journal of Occupational Therapy, 61, 704-709. doi:10.5014/ajot.61.6.704

Johnston, J. L., Adams, R., \& Helfrich, C. A. (2001). Knowledge and attitudes of occupational therapy practitioners regarding wife abuse. Occupational Therapy in Mental Health, 16, 35-52. doi:10.1300/J004v16n03 03

Katz, L. F., Hunter, E., \& Klowden, A. (2008). Intimate partner violence and children's reaction to peer provocation: The moderating role of emotion coaching. Journal of Family Psychology, 22, 614-621. doi:10.1037/a0012793

Kiresuk, T. J., \& Sherman, R. E., (1968) Goal attainment scaling: A general method for evaluating comprehensive community mental health programs. Community Mental Health Journal, 4, 443-453. doi:10.1007/BF01530764

Kimerling, R., Alvarez, J., Pavao, J., Mack, K. P., Smith, M. W., \& Baumrind, N. (2009). Unemployment among women: Examining the relationship of physical and psychological intimate partner violence and posttraumatic stress disorder. Journal of Interpersonal Violence, 24, 450-463. doi:10.1177/0886260508317191

Krane, J., \& Davies, L. (2007). Mothering under difficult circumstances: Challenges to working with battered women. Journal of Women \& Social Work, 22, 23-38.

Krug, E. G., Dahlberg, L. L., Mercy, J. A., Zwi, A. B. \& Lozano, R., (Eds.) (2002). World report on violence and health. Geneva: World Health Organization.

Lang, J. M., \& Stover, C. S. (2008). Symptom patterns among youth exposed to intimate partner violence. Journal of Family Violence, 23, 619-629. doi:10.1007/s10896-008-9184-5

Law, M. C. (Ed.). (2008). Evidence-based rehabilitation: A guide to practice (2nd ed.). Thorofare, NJ: Slake.

McFarlane, J. \& Parker, B. (1994). Abuse during pregnancy. A protocol for prevention and intervention. New York: National March of Dimes Birth Defects Foundation.

McFarlane, J., Malecha, A., Gist, J., Watson, K., Batten, E., Hall, I., et al. (2002). An intervention to increase safety behaviors of abused women: Results of a randomized clinical trial. Nursing Research, 51, 347-354. doi:10.1097/00006199-200211000-00002

Mcnutt, L., Carlson, B. E., Persaud, M., \& Postmus, J. (2002). Cumulative abuse experiences, physical health and health behaviors. Annals of Epidemiology, 12, 123-130. doi:10.1016/S1047-2797(01)00243-5

Mechanic, M. B., Weaver, T. L., \& Resick, P. A. (2008). Risk factors for physical injury among help-seeking battered women: An exploration of multiple abuse dimensions. Violence against Women, 14, 11481165. doi:10.1177/1077801208323792
Olivas-De La O, T. (2008). Perspectives. OT saved my life: Surviving domestic violence. OT Practice, 13, 23-24.

Ottenbacher, K. J., \& Cusick, A. (1993). Discriminative versus evaluative assessment: Some observations on goal attainment scaling. American Journal of Occupational Therapy, 47, 349-354.

Plichta, S. B. (2004). Intimate partner violence and physical health consequences: Policy and practice implications. Journal of Interpersonal Violence, 19, 1296-1323. doi:10.1177/0886260504269685

Public Health Agency of Canada. (2009). What is intimate partner violence. URL (last checked 10 December 2011).

http://www.phac-aspc.gc.ca/ncfv-cnivf/faqs/fem-initim-partnr-abus-e ng.php

Siebes, R. C., Ketelaar, M., Gorter, J. W., Wijnroks, L., De Blécourt, A., Reinders-Messelink, H. et al. (2007). Transparency and tuning of rehabilitation care for children with cerebral palsy: A multiple case study in five children with complex needs. Developmental Neurorehabilitation, 10, 193-204. doi:10.1080/13638490601104405

Spangaro, J. M., Zwi, A. B., Poulos, R. G., \& Man, W. Y. (2010) Who tells and what happened: Disclosure and health service responses to screening for intimate partner violence. Health and Social Care in the Community, 18, 671-680. doi:10.1111/j.1365-2524.2010.00943.x

Statistic Canada. (2005). Family violence in Canada: A statistical profile, 2005 (No. 85-224-XIE). URL (last checked 10 December 2011). http://www.statcan.gc.ca/pub/85-224-x/85-224-x2005000-eng.pdf

Statistic Canada. (1994). Wife assault: The findings of a national survey. URL (last checked 10 December 2011). http://www.phac-aspc.gc.ca/ncfv-cnivf/publications/femnational-eng. php

Statistic Canada. (2008). Family violence in Canada: A statistical profile, 2008 (No. 85-224-X). URL (last checked 12 November 2011). http://www.statcan.gc.ca/pub/85-224-x/85-224-x2008000-eng.pdf

Suglia, S. F., Enlow, M. B., Kullowatz, A., \& Wright, R. J. (2009). Maternal intimate partner violence and increased asthma incidence in children: Buffering effects of supportive caregiving. Archives of Pediatrics \& Adolescent Medicine, 163, 244-250. doi:10.1001/archpediatrics.2008.555

Taylor, C. A., Guterman, N. B., Lee, S. J., \& Rathouz, P. J. (2009). Intimate partner violence, maternal stress, nativity, and risk for maternal maltreatment of young children. American Journal of Public Health, 99, 175-183. doi:10.2105/AJPH.2007.126722

Wathen, C. N., \& MacMillan, H. L. (2003). Interventions for violence against women: Scientific review. The Journal of the American Medical Association, 289, 589-600. doi:10.1001/jama.289.5.589

Woods, S. J., Hall, R. J., Campbell, J. C., \& Angott, D. M. (2008). Physical health and posttraumatic stress disorder symptoms in women experiencing intimate partner violence. Journal of Midwifery and Women's Health, 53, 538-546. doi:10.1016/j.jmwh.2008.07.004

Wuest, J., Merritt-Gray, M., Ford-Gilboe, M., Lent, B., Varcoe, C., \& Campbell, J. C. (2008). Chronic pain in women survivors of intimate partner violence. Journal of Pain, 9, 1049-1057. doi:10.1016/j.jpain.2008.06.009 